\title{
INFLUENCE OF THE BUILT ENVIRONMENT ON PATIENT SAFETY AND WELL-BEING: A FUNCTIONAL PERSPECTIVE
}

\author{
Natália Ransolin ${ }^{1}$, Tarcísio Abreu Saurin², and Carlos Torres Formoso ${ }^{3}$
}

\begin{abstract}
In healthcare facilities, patient safety and well-being are known to be influenced by the built environment (BE). However, mechanisms linking BE to patient safety and wellbeing are not well-understood, which hinders the prevention and mitigation of undesired outcomes. In this paper, the Functional Resonance Analysis Method (FRAM) is used to model the functions carried out by caregivers and patients in an Intensive Care Unit (ICU), supporting the analysis of how variability in meeting BE requirements propagates across the work system and impacts patient safety and well-being. The FRAM model was based on observations of everyday work in the ICU, interviews with 24 professionals, and analysis of documents. One scenario derived from the FRAM model is discussed, emphasizing impacts on patient safety and well-being. Results show the utility of understanding how healthcare services work under real circumnstances, as a basis for BE design. Findings also indicate that the variability of everyday healthcare services may either amplify or dampen the impacts of unfulfilled BE requirements on patient safety and well-being.
\end{abstract}

\section{KEYWORDS}

Built Environment, Requirements Management, FRAM, Patient Safety, Patient WellBeing

\section{INTRODUCTION}

The design of the built environment (BE) must account for many types of requirements from several stakeholders (Kim et al., 2015). Requirements express the properties that a building must have in order to meet stakeholders' needs (Kamara et al., 1999). In this study, BE requirements have been defined as building attributes, including components, utilities, and spaces, that should be in place so that the BE fulfill the needs of end users i.e. people that use the building as patients, visitors, and permanent or temporary work.

In healthcare facilities, the main end user is the patient, whose requirements therefore have a high priority (Tzortzopoulos et al., 2009). Thus, understanding the activities

1 PhD Student, Civil Engineering Post-Graduation Program: Construction and Infrastructure, Federal University of Rio Grande do Sul (UFRGS), Porto Alegre, Brazil, natalia.ransolin@ufrgs.br, orcid.org/0000-0002-7128-8000

2 Professor, Industrial Engineering Post-Graduation Program, Federal University of Rio Grande do Sul (UFRGS), Porto Alegre, Brazil, saurin@ufrgs.br, orcid.org/0000-0003-2929-5888

3 Professor, Civil Engineering Post-Graduation Program: Construction and Infrastructure, Federal University of Rio Grande do Sul (UFRGS), Porto Alegre, Brazil, formoso@ufrgs.br, orcid.org/0000$\underline{0002-4772-3746}$ 
performed in healthcare facilities, especially those directly or closely related to patient care, is necessary for identifying patients' requirements (Rosso and Saurin, 2018). In a high abstraction level, these requirements may be interpreted as patient safety and wellbeing (Lucas et al., 2012), which are influenced by BE (Ulrich et al., 2008; Pickup, 2018). $\mathrm{BE}$ design decisions that benefit patient safety and well-being are logically connected to the lean construction principles (Koskela, 2000) concerned with value generation from the customer viewpoint (e.g. patient) and flow of activities (e.g. healthcare services).

The analysis of how a system functions should emphasize work-as-done, which corresponds to what people actually do in the workplace (Hollnagel, 2012). This is in contrast to work-as-imagined, which is described in regulations, policies, and protocols (Hollnagel et al., 2014). However, BE design approaches commonly take for granted that work-as-done must be equal to work-as-imagined (Kim and Fisher, 2014), which is in conflict with the dynamic nature of healthcare (Hollnagel et al., 2014).

In this study, the Functional Resonance Analysis Method (FRAM), proposed by Hollnagel (2012), has been adopted for modelling interactions between BE and patient safety and well-being. The study is based on the assumption that BE impacts on patient safety and well-being do not necessarily occur directly and linearly, rather being moderated by the functions carried in the workspace. In FRAM, a function corresponds to the activities required to produce a certain outcome, being described by six aspects: input, output, resources, preconditions, control, and time (Hollnagel, 2012). The precondition aspect of FRAM functions is particularly relevant for this investigation, because it accounts for conditions that must exist before carrying out a function. While a precondition does not start a function, it should be in place before that (Hollnagel, 2012). This definition of precondition is similar to the concept of requirements, which therefore indicates the possibility of considering BE requirements when developing a FRAM model (Ransolin et al., 2020). The applicability of this approach is demonstrated through the analysis of a scenario in an adult Intensive Care Unit (ICU).

Earlier studies have adopted FRAM in lean construction, but only in the context of safety management (e.g. Saurin, 2016). Those studies have indicated the usefulness of FRAM for understanding variability propagation and accounting for the complexity of construction projects, which have been traditional topics of interest in lean construction. For the purpose of this study, variability is created by unfulfilled BE requirements and it propagates across the system through functions carried out by caregivers and patients. The study reported in this paper is part of a broader research project carried out at the same ICU, in which a framework for the integrated modelling of BE and other functional requirements was applied (Ransolin et al., 2020). That study relied on the combined use of FRAM and Building Information Modelling for requirements management.

\section{BACKGROUND}

\section{Built ENVIRonment ImPaCts ON PATIENT SAFETY AND Well-Being}

Healthcare facilities support both functional and emotional needs of patients and caregivers. Value for these stakeholders can be tangible, such as the avoidance of medical errors and falls, or intangible, such as environmental comfort, satisfaction, and efficiency (Zhang et al., 2016). However, healthcare facilities are often physically and psychologically stressful for patients, their next-of-kin, and employees (Intensive Care Society, 2016; La Calle et al., 2017). 
Some BE conditions that impact patient safety and well-being, both in ICUs and other hospital settings, include noise, light, privacy, comfort, views of plants and sunlight, access to the external environment, and accessibility (Ulrich et al., 2008; Rybkowski, 2009; Hicks et al., 2015; Pickup, 2018). Unpleasant environments have been associated with higher medication dosage to cope with discomfort, anxiety, pain, longer hospital stay, and staff dissatisfaction (Rashid, 2010). Furthermore, inadequate layout of workspaces and outdated or difficult access to information are known as contributing factors to errors in health services (Chaudhury et al., 2009).

The discipline that addresses good practices for designing the $\mathrm{BE}$ of healthcare facilities is known as Evidence Based Design (EBD). EBD supports BE designers' and managers' decision-making so as they account for evidence available in the literature (Ulrich et al., 2008; Rybkowski, 2009). An example of good practice reported in the literature is the provision of stress-reducing music or sunlight, which can decrease the need for giving drugs to patients (Rybkowski, 2009; Zhang et al., 2017). Therefore, EBD is useful for generating value to stakeholders, which is a lean construction principle (Zhang et al., 2016). However, it is difficult to carry out controlled studies for producing highly credible evidence (Rybkowski, 2009), due to the large number of variables that play a role in the performance of healthcare services and patient outcomes.

\section{PRINCIPLES OF FRAM}

FRAM is an approach for modelling variability propagation in socio-technical systems, which are the ones in which people interact with technical and managerial systems, all subjected to the influence of the external environment (Hendrick and Kleiner, 2001). As such, FRAM provides both a descriptive and visual representation of how a function or sequence of functions generally occur (Hollnagel, 2012). FRAM is based on four principles (Hollnagel et al., 2014):

(i) Equivalence of successes and failures: it assumes that variability is underlying both desired and undesired outcomes. What makes the difference in outcomes is the specific combination of variabilities in a certain moment in time;

(ii) Approximate adjustments: adjustments of performance are always necessary, whether due to finite resources and time, or underspecified plans. However, these performance adjustments are not perfect, and therefore variability is part of everyday work in complex systems;

(iii) Emergence: it acknowledges that not all results can be explained as having a specific and identifiable cause. Moreover, variability emerging from each of the multiple functions performed can combine unexpectedly, producing a non-linear effect; and

(iv) Functional resonance: it is a manifestation of the unintended interaction of the normal variability of each function in a system.

A FRAM model consists of interconnected functions, their corresponding variabilities, and couplings between functions (Hollnagel et al., 2014). The first step when developing a FRAM model is to describe the functions that make up the system, according to six aspects (Hollnagel, 2012):

- Input (I): corresponds to what the function processes or transforms or what starts the function;

- Output (O): result from the function, either an entity or a state change;

- Preconditions $(\mathrm{P})$ : conditions that must be exist before a function can be carried out; 
- Resources $(\mathrm{R})$ : what a function consumes to produce the output;

- Time (T): temporal constraints affecting the function (with regard to starting time, finishing time or duration); and

- Control (C): how the function is monitored or controlled.

Next, an analysis of the variability of the outputs should be conducted, both in terms of time (it can be too early, on-time, too late, or not at all) and precision (it can imprecise, acceptable, or precise). Then, the couplings between functions should be established, modelling how the outputs are connected to the other aspects - this indicates the pathway for variability propagation across the system. A visual representation of the elements of FRAM models is represented in Figure 1.

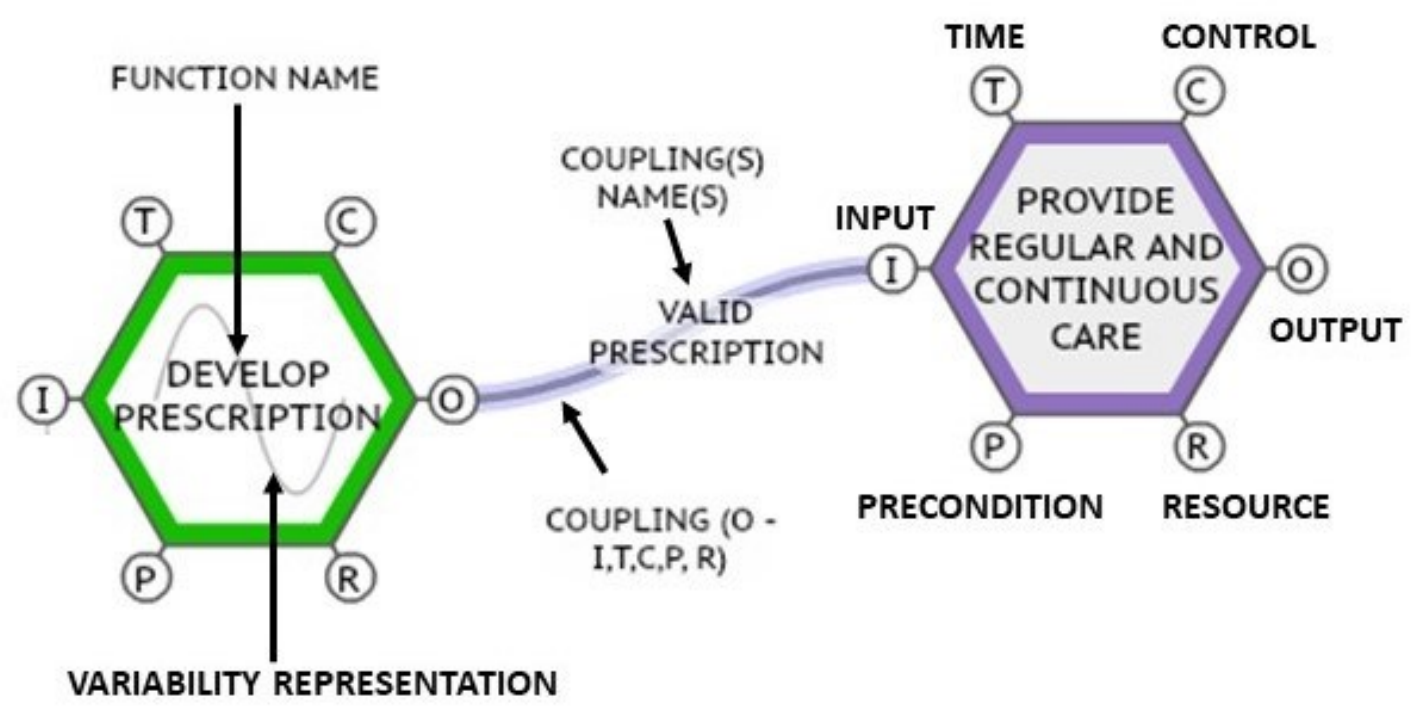

Figure 1: Visual representation of the elements of a FRAM model.

\section{RESEARCH METHOD}

Three main sources of data were used to identify BE requirements of the ICU and to develop the FRAM model: interviews, observation of functions conducted by caregivers, and analysis of regulations. As for interviews, these involved: 24 professionals, including managers and workers; two members of patient families, and one patient - this was interviewed after being discharged from the ICU. A script of 12 questions guided the interviews (e.g. how does the BE facilitate or hinder the development of your activities?), focusing on the functions performed by the interviewees and the relationships of those functions with the BE. All participants signed a term of informed consent before being interviewed. The study was approved by the hospital's ethical committee.

In turn, two Brazilian regulations were analysed: one of them addressed the functioning of healthcare services (Anvisa, 2010), and the other related to $\mathrm{BE}$ requirements in hospitals (Anvisa, 2002). Altogether, data collection took 85 hours, being 62 hours for interviews and 23 hours for observations.

Data from the aforementioned sources was subject to a content analysis with predefined themes associated with the information needed for developing a FRAM model that could represent the ICU everyday functioning. The themes were: functions; their aspects (input - I, output - O, precondition - P, resources - R, control - C, and time - T); 
and output variability for each function. As previously mentioned, all BE requirements were regarded as a type of precondition aspect of functions.

Based on this information, the research team devised the first version of the FRAM model. A key assumption of this model was that there would be two functions defining the model boundaries: < fulfil BE requirements $>$, which provided preconditions and other aspects to the functions carried out by caregivers; and <receive or respond to care interventions $>$, which was performed by patients. Variability of the output of this latter function was interpreted as a manifestation of detrimental impacts on patient safety and well-being.

This draft of the FRAM model was then discussed in 11 face-to-face meetings with doctors, physiotherapist, nurses, and nurse technicians. After a final model was produced, researchers and professionals jointly identified possible scenarios of functional resonance that met two criteria: there should be risks to patient safety and well-being, and the unfulfillment of BE requirements should be a contributing factor to those risks. One of these scenarios is presented in this paper. This functional resonance scenario is based on the generic FRAM model, but slightly different from it. The main difference relates to the inclusion of functions that display resilient performance in action - i.e. functions that only existed because resilience was necessary to cope with unfulfilled BE requirements. These functions played a key moderating role between the BE and patient safety and wellbeing.

\section{RESULTS}

Figure 2 shows the selected functional resonance scenario that illustrates BE impacts on patient safety and well-being. During the first care assessment, the patient may not be able to convey accurate information on their health condition, as ICU patients often arrive distressed. Then, it is important to fulfil BE requirements such as "cozy environment" and "space to accommodate visitors in the patient bay", as these may improve the performance of the function <communicate with family>. If these requirements are unfulfilled, that communication may be imprecise and generate a variability that propagates to $<$ carry out interdisciplinary round $>$, in which a multidisciplinary care team discusses the patient clinical conditions on a daily basis. Hence, decision-making made by that care team may be hindered by innacurate information on the patient clinical history, posing patient safety hazards. Furthermore, an uncomfortable environment for family members and visitors may affect the experience of patients, who may feel guilty about their loved ones. Thus, the BE requirement "space to accommodate visitors in the patient bay" is important for the well-being of patients and their families. 


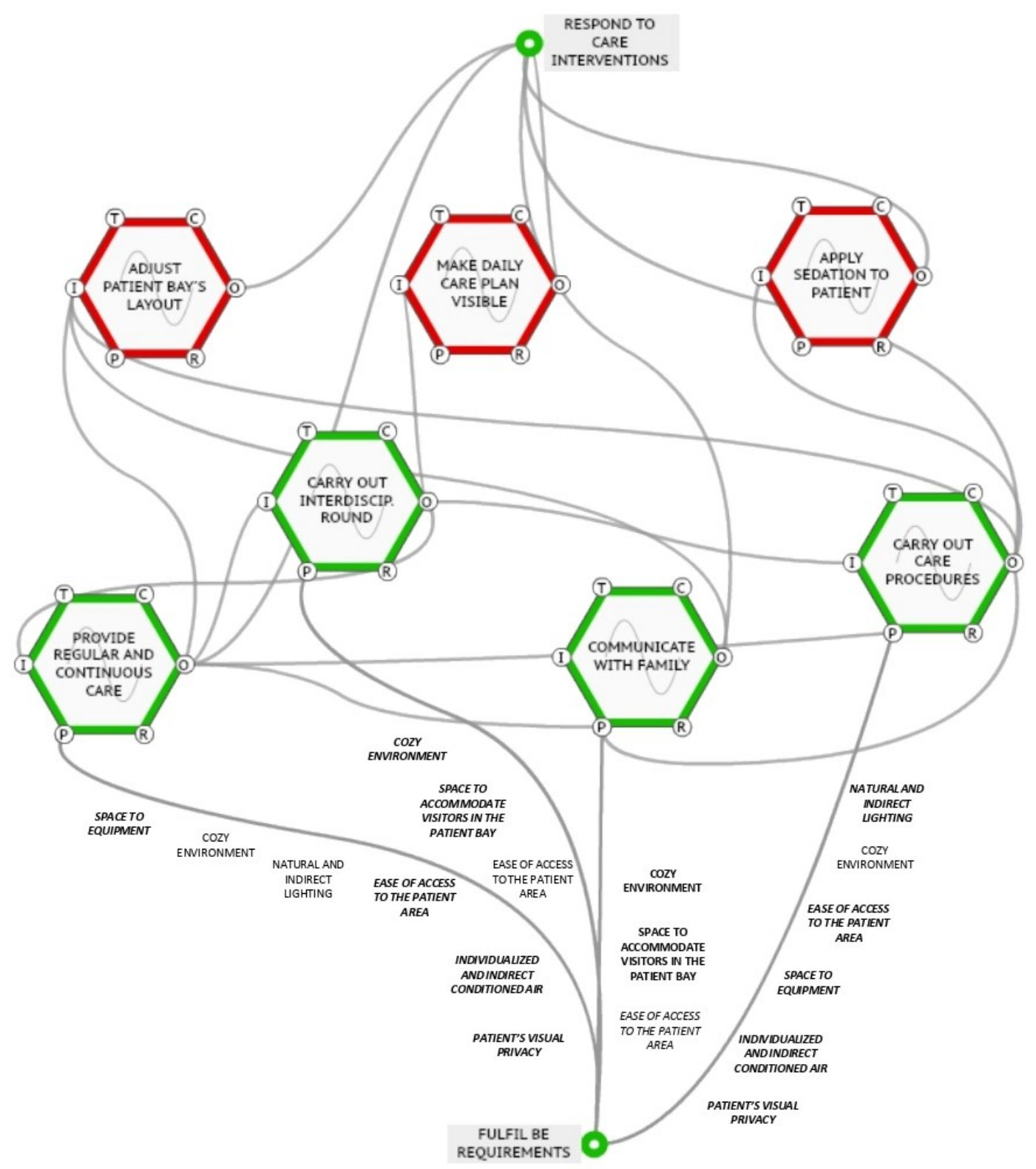

Notes: hexagons with green borders represent care functions; red borders represent functions that display resilient performance; functions in rectangles are the boundaries of the system. The bold and italic requirements are those in which the unfulfillment reaches the resilient performance functions.

Figure 2: Functional resonance scenario: influence of the BE on patient safety and wellbeing.

In addition, <carry out care procedures $>$ may occur during visiting hours, and therefore patients and family members from the surrounding beds may view and listen to the entire procedure. As a result, patients from other beds can be frightened by imagining themselves at the same situation (e.g. tracheostomy), thus impacting patient wellbeing.

The functions $<$ provide regular and continuous care $>$ and $<$ carry out care procedures $>$ also need BE requirements such as "ease of access to the patient area" and "space to 
equipment". Due to the configuration of the bay, caregivers do not have $360^{\circ}$ access around the bedside. Resilient performance is thus necessary to cope with that drawback. This involves changing the bay's layout for each clinical intervention, which is represented in Figure 2 by the function <adjust patient bay's layout>. As a drawback, these layout changes cause discomfort to the patient and increase the risk of accidents. These changes may also have implications for patients from neighbouring beds, which may have their privacy compromised, thus hindering the BE requirement "patient's visual privacy", which is related to well-being.

After receiving care procedures, a patient's awakening should be as peaceful as possible. Each patient has a body temperature comfort, which varies due to clinical conditions. However, the BE requirement "individualized and indirect air conditioned" is unfulfilled in the patient bay, where there is only one air exit above the bed - furthermore, all bays have the same temperature.

Lighting is also an important factor for patient comfort. It must allow easy visualization by care teams during the function < carry out care procedures $>$. The lighting system must also make intensity and direction adjustments possible according to patient needs, in order to meet the requirement "natural and indirect lighting" - this is unfulfilled at the ICU. Also, natural light does not reach all patient bays and the ceiling lighting has a mirrored surface. It can frighten patients when they wake up and see themselves reflected on the lamps. These factors contribute to delirium, which is health condition that occurs when patients lose contact with the outside and natural lighting.

A resilient strategy for coping with this situation, when the patient wakes up agitated, is represented in Figure 2 by the function <apply sedation to patient $>$. However, sedation might increase delirium, as the patient becomes more and more unaware of time and space. In turn, this influences patient length of stay, causing unnecessary exposure to ICU conditions.

Another example of resilient strategy refers to the function <make daily care plan visible $>$. This plan, which guides care provision for the next 24 hours, is developed after the daily interdisciplinary round. Early on the implementation of the multidisciplinary rounds, which is a recent initiative at the studied ICU, nurse technicians and allied health professionals only had access to the clinical decisions by directly contacting doctors. In order to improve access to clinical information, a solution was devised by nurse technicians: posting the daily care plan on a support desk at the patient's bedside.

\section{DISCUSSION}

According to the presented scenario, variability in fulfilling BE requirements propagates across space and time through everyday functions of caregivers, hindering patient safety and well-being. Results also pointed out the need for understanding how employees cope with unfulfilled BE requirements, displaying resilient performance. However, resilience may imply in the creation of new functions, hence increasing opportunities for unwanted interactions. This has been illustrated by the function <adjust patient bay's layout>, which brings its own unintended consequences and only exists because of unfulfilled BE requirements.

Traditional BE design management practices usually disregard work-as-done and its unavoidable variability, an inherent feature of complex systems. Therefore, it is necessary to develop new design management approaches that take into account the complexity of healthcare. FRAM is useful for that purpose, by supporting the joint modelling of BE requirements and work-as-done by caregivers and patients. Thus, the approach adopted 
in this study can contribute to value generation through supporting the fulfillment of BE requirements that impact patient safety and well-being. It is also worth noting that this type of investigation can strengthen the EBD literature by acknowledging that variability is to some extent unavoidable in healthcare services, and thus an one size fits-all evidence is elusive.

\section{CONCLUSIONS}

This paper has discussed the use of FRAM for understanding the interactions between $\mathrm{BE}$ and patient safety and well-being, which has been defined as a high level requirement of clients of healthcare facilities. The adopted approach has a number of implications, namely: (i) it sheds light on the gap between work-as-done and work-as-imagined, making clear that BE designers need to account for the variability of every day work and resilient performance when identifying requirements and developing design solutions; (ii) it provides a logic and traceable approach for connecting BE requirements with functions, thus providing a new tool for requirements management; and (iii) it indicates that unfulfillment of BE requirements has implications to spaces and functions across the whole work system. Furthermore, the adopted approach is useful for the EBD literature, by offering a means for mapping $\mathrm{BE}$ design solutions onto patient safety and well-being.

Some limitations of this study must be mentioned: (i) it was developed in a specific type of healthcare service (i.e. ICU), and thus the adopted approach needs to be tested in other contexts; (ii) the perspective of patients and their families was not explored in depth, due to the low number of interviewees from those groups; and (iii) the full implications of the functions that display resilient performance have not been assessed. In this respect, an opportunity for future studies is the deeper investigation of those functions, assessing the extent to which they are unavoidable and whether they can be supported by BE design.

\section{REFERENCES}

Anvisa - Agência Nacional de Vigilância Sanitária (National Health Surveillance Agency). 2002. Resolução de Diretoria Colegiada (Collegiate Board Resolution) RDC N.50. Brazil.

Anvisa - Agência Nacional de Vigilância Sanitária (National Health Surveillance Agency). 2010. Resolução de Diretoria Colegiada (Collegiate Board Resolution) RDC N.7. Brazil.

Chaudhury, H., Mahmood, A., and Valente, M. 2009. "The effect of environmental design on reducing nursing errors and increasing efficiency in acute care settings: A review and analysis of the literature." Environment and Behavior, 41(6), 755-786.

Hendrick, H. W., \& Kleiner, B. M. 2001. "Macroergonomics: An introduction to work system design". Human Factors and Ergonomics Society.

Hicks, C., McGovern, T., Prior, G., and Smith, I. 2015. "Applying lean principles to the design of healthcare facilities." Int. J. of Production Economics, 170, 677-686.

Hollnagel, E. 2012. FRAM: the Functional Resonance Analysis Method: Modeling complex socio-technical systems. Ashgate, Burlington.

Hollnagel, E., Hounsgaard, J., Colligan, L. 2014. FRAM - The Functional Resonance Analysis Method - a handbook for the practical use of the method. Centre for Quality, Region of Southern Denmark.

Intensive Care Society. 2016. Guidelines for the provision of intensive care services. $<\mathrm{https}$ ://www.ficm.ac.uk/sites/default/files/gpics_ed.1.1_-_2016__final_with_covers.pdf $>(\operatorname{Mar} 20,2020)$. 
Kamara, J.M., Anumba, C.J., and Evbuomwan, N.F. 1999. "Client requirements processing in construction: a new approach using QFD." Journal of architectural engineering, 5(1), 8-15.

Kim, T.W. and Fischer, M. 2014. "Ontology for representing building users activities in space-use analysis." J. Construction Engin. and Manage., 140(8), 04014035.

Kim, T.W., Kim, Y., Cha, S.H., and Fischer, M. 2015. "Automated updating of space design requirements connecting user activities and space types." Automation in Construction, 50, 102-110.

Koskela, L. 2000. An exploration towards a production theory and its application to construction. VTT Technical Research Centre of Finland.

La Calle, G.H., Martin, M.C., and Nin, N. 2017. "Seeking to humanize intensive care." Revista Brasileira de terapia intensiva, 29(1), 9-13.

Lucas, J.D. 2012. "An integrated BIM framework to support facility management in healthcare environments." Doctoral dissertation, Virginia Tech.

Pickup, L., Lang, A., Atkinson, S., and Sharples, S. 2018. "The dichotomy of the application of a systems approach in UK healthcare the challenges and priorities for implementation." Ergonomics, 61(1), 15-25.

Ransolin, N., Saurin, T.A., and Formoso, C. T. 2020. "Integrated modelling of built environment and functional requirements: implications for resilience." Applied Ergonomics, 88, 103154.

Rashid, M. 2010. "Environmental design for patient families in intensive care units." Journal of Healthcare Engineering, 1(3), 367-398.

Rosso, C.B., and Saurin, T.A. 2018. "The joint use of resilience engineering and lean production for work system design: A study in healthcare." Applied Ergonomics, 71, $45-56$.

Rybkowski, Z.K. 2009. "The application of root cause analysis and target value design to evidence-based design in the capital planning of healthcare facilities." Doctoral Dissertation, University of California, Berkeley.

Saurin, T.A. 2016. "The FRAM as a Tool for Modelling Variability Propagation in Lean Construction." Proc. 24 th Annual Conference of the International Group for Lean Construction, Boston, Mass., USA, v. 2. p. 3-12.

Tzortzopoulos, P., Codinhoto, R., Kagioglou, M., Rooke, J.A., and Koskela, L.J. 2009. "The gaps between healthcare service and building design: a state of the art review." Ambiente Construído, 9(2), 47-55.

Ulrich, R. S., Zimring, C., Zhu, X., DuBose, J., Seo, H.B., Choi, Y. S., and Joseph, A. 2008. "A review of the research literature on evidence-based healthcare design." Health Environments Research \& Design J., 1, 61-125.

Zhang, Y., Tzortzopoulos, P., and Kagioglou, M. 2016. "Evidence-based design in healthcare: A lean perspective with an emphasis on value generation." In Proceedings of the 24th Annual Conference of the International Group for Lean Construction (IGLC), Boston, MA, USA. v.1. p. 20-22.

Zhang, Y., Tzortzopoulos, P., and Kagioglou, M. 2017. "How evidence-based design supports better value generation for end-users." In: Proc. $25^{\text {th }}$ Ann. Conf. Int. Group for Lean Construction, 9-12 July, Heraklion, Greece. v.1. p. 579-586. 
Influence of the Built Environment on Patient Safety and Well-being: a Functional Perspective

This page was intentionally left blank. 
Natália Ransolin, Tarcísio Abreu Saurin, and Carlos Torres Formoso

This page was intentionally left blank. 
Influence of the Built Environment on Patient Safety and Well-being: a Functional Perspective

This page was intentionally left blank. 